\title{
Total Hip Arthroplasty in Neglected Developmental Dysplasia Hip, a Case Report
}

\author{
Tan Wei How, Wan Hazmy Che Hon \\ KPJ Healthcare University College, Nilai, Malaysia \\ Email: tanweihow@yahoo.com.my
}

How to cite this paper: How, T.W. and Hon, W.H.C. (2021) Total Hip Arthroplasty in Neglected Developmental Dysplasia Hip, a Case Report. Journal of Biosciences and Medicines, 9, 1-5.

https://doi.org/10.4236/jbm.2021.97001

Received: May 5, 2021

Accepted: June 25, 2021

Published: June 28, 2021

Copyright $\odot 2021$ by author(s) and Scientific Research Publishing Inc. This work is licensed under the Creative Commons Attribution International License (CC BY 4.0).

http://creativecommons.org/licenses/by/4.0/

\begin{abstract}
Developmental dysplasia of hip is a disorder of abnormal development of the hip joint resulting early osteoarthritis of the hip. In late stage of disease, total hip arthroplasty is the option to reduce pain. Total hip arthroplasty in development dyplastic hip is a very challenging procedure in view of abnormal anatomy and biomechanic of the hip. A proper pre-operative planning is important of successful surgery. We report a case of a 60 -year-old woman with neglected DDH with severe hip pain, who underwent total hip replacement with good result.
\end{abstract}

\section{Keywords}

Total Hip Arthroplasty, Developmental Dysplasia Hip, Neglected

\section{Introduction}

Developmental dysplasia of the hip (DDH) is a disorder of abnormal development of the hip joint resulting in a shallow acetabulum with lack of anterior and lateral coverage. NY Boo et al. reported incidence of developmental dysplasia of the hip in Malaysia varies from 0.7/1000 live births in normal cephalic deliveries and 10.7/1000 live births in breech deliveries [1]. The risk factors are breech presentation, female sex $(2-4 \mathrm{x}$ higher than male), primiparity and family history of DDH.

DDH resulting in joint pain and destruction of the articular cartilage, develop secondary osteoarthritis. Various conservative and operative options are available for treatment of DDH. In early stage, conservative management including weight loss, lifestyle modification, joint injection and physiotherapy with the aim to relieve pain and delay the progression of joint destruction. For advanced stage of painful hips, total hip arthroplasty is the method. There is estimated that $10 \%$ 
of all total hip arthroplasty are performed as a result of hip dysplasia and $48 \%$ were less than 50 years old.

Anatomy of DDH usually significantly altered. The abnormalities seen in acetabulum, femur and soft tissues. An acetabulum with dysplasia can be shallow, narrow and lateralized due to increase anteversion and deficiency of anterior and superior walls of acetabulum [2]. As a result of these deformities, the femoral head tends to dislocated anteriorly, laterally and superiorly to the true acetabulum on the ilium to form false acetabulum. The dysplastic femur has a small femoral head and femoral neck is shortened and anteversion with increase neck-shaft angle [3]. Narrowing of medullary canal around the level of lesser trochanteric is seen in Crowe IV DH [4]. The abductor muscles orientation is more transverse compared to normal hip. The hamstrings, adductor and rectus femoris muscles are shortened. Neurovascular is in shorten, which might injury in acute lengthening.

There are 2 classifications used to describe DDH which are Hartofilakidis and Crowe classification. There are 3 gradings for Hartofilakidis. The femoral head is covered within the true acetabulum in the first stage. Second stage called low dislocation where the femoral head creates a false acetabulum superior to the true acetabulum. There is complete absence of the superior wall. Inadequate depth of the true acetabulum. Third stage also known as high dislocation where the femoral head is completely uncovered by the true acetabulum and migrated superiorly and posteriorly. There is a complete deficiency of the acetabulum and excessive anteversion of the true acetabulum. Whereas, there are four gradings in Crowe classification, depending on the proximal migration of head neck junction from the inter-teardrop line. Grade I if the proximal migration less than $50 \%$. Grade II 50 to $75 \%$. Grade III 75 to $100 \%$ and grade IV if more than $100 \%$.

\section{Case Report}

60 year old woman, presented with history of limping since childhood and right hip pain for 2 years. She denied any history of trauma or hip injury. She has to take pain medications on daily basic. The pain worsens over past 3 months and her daily activities were affected by the hip pain. Her pain score was $8 / 10$. On examination, the patient was walking with waddling gait. Extension, adduction and rotational movement of the right hip were painfully restricted. 15 degree flexion deformity and 10 degree abduction deformity were present. Right lower limb is $2 \mathrm{~cm}$ shorter than left lower limb. $\mathrm{X}$ ray pelvic (Figure 1(a) and Figure 1(b)) showed avascular necrosis of right femoral head with osteoarthritis of right acetabulum. The acetabulum was shallow and femoral head subluxated compare to the left acetabulum (Crowe Type 1). After failed conservative management, she decided to undergo total hip arthroplasty.

Intra-operatively, she was put on general anaesthesia with muscle relaxant. She was in left lateral position. Lateral approach done. Soft tissues disecting till bone. Femoral neck cut and femoral head removed. From the pre-operative $\mathrm{x}$ 
ray, the femoral head minimally subluxated superiorly about $2 \mathrm{~cm}$ compared to the left and the bone stock for acetabulum still good. We decided to medialise the acetabulum cup to increase the bone implant interface. We used uncemented acetabulum cup with screw augmentation. Acetabulum cup was stable with good bone implant coverage. During trial implant reduction, the hip joint was not able to reduce. After a few trial of reduction, there was split fracture over intertrochanteric of the femur. Circlage wire used to reduce the fracture and cemented femoral stem inserted. After soft tissue release done, the hip was able to reduce and stable in all direction except in extreme external rotation. She was put on abduction pillow post operatively.

Post operative X ray (Figure 2(a) and Figure 2(b)) showed intertrochanteric fracture well reduced with circlage wire and good acetabular cup well implanted with bone. She was discharge well after 3 days post-operative. She was advised for wheel chair ambulation for 6 week then partial weight bearing with walking frame. She was able to walk independently after 6 months.

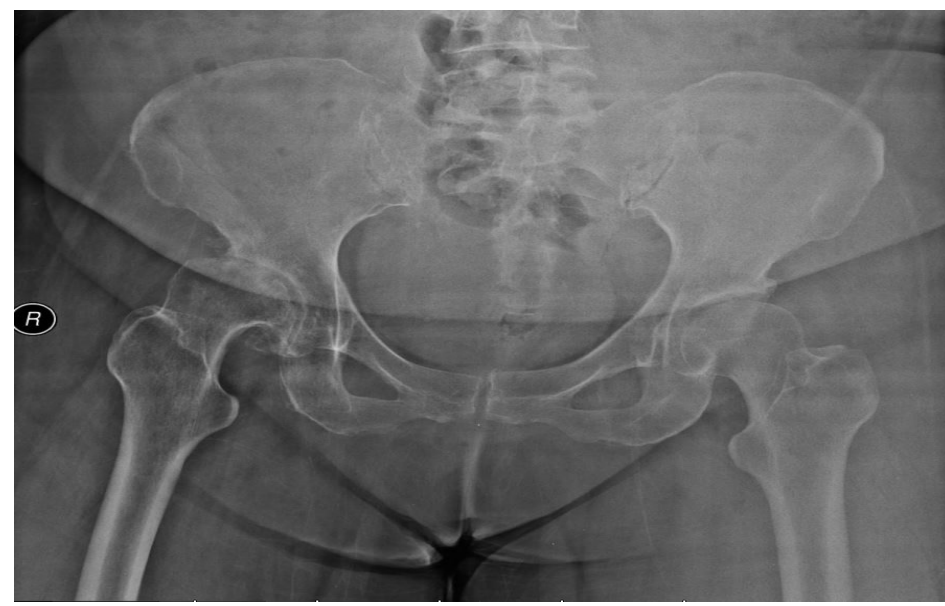

(a)

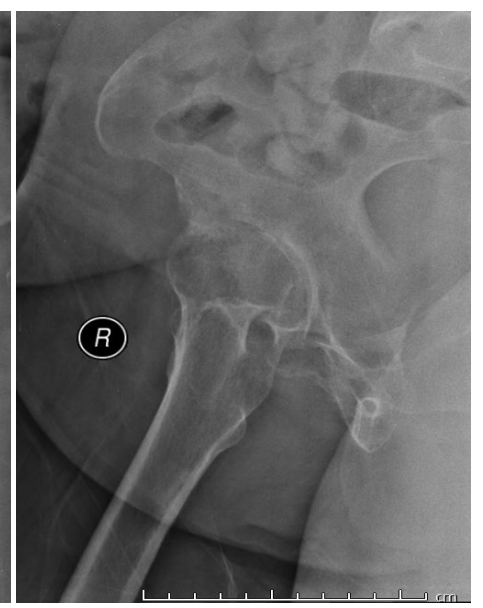

(b)

Figure 1. Pre-operative pelvic X ray showed avascular necrosis of right femoral head with osteoarthritis of right acetabulum.



(a)



(b)

Figure 2. Post-operative right hip X ray. 


\section{Discussion}

In view of abnormal anatomy and biomechanic of hip in $\mathrm{DDH}$, there is major challenge in doing total hip arthroplasty on these patients due to deficient in acetabular bone stock, abnormal femoral anatomy with increased neck shaft angle and abnormal valgus deformation, increase anteversion, musle contracture and limb-length discrepancy. Pre-operative evaluation played important role. Hip range of motion and limb length shortening must be measured. Pelvic and hip $\mathrm{X}$ ray needed for classification and determined the deformities. Judet view might need to determine the acetabular bone stock. CT scan can be useful to evaluate the acetabular bone stock and femoral version [5]. The acetabular bone stock is important to determine the implantation of acetabular cup. Bone grafting and reconsctruction systems might required in low acetabular bone stock patient.

There are 2 main aims in acetabulum reconstruction. First is the placing of acetabular component to the true acetabular to provide normal biomechanical properties of hip with normal center of rotation. Second is the coverage of the acetabular cup by the acetabular bone to maximise the bone implant interface to prevent early mechanical failure. Total of $60 \%$ to $70 \%$ coverage of cementless acetabular cup is acceptable [6]. In low dislocation case, there is less bone stock over the lateral part of the acetabulum resulting to less bone support for acetabulum component. There is several surgical technique to improve the coverage of acetabular component. Acetabuloplasty or medialization of acetabular cup can improve on the coverage of bone and reduce the joint reactive forces but if still inadequate (usually the superolateral part), acetabular augmentation of bone graft and cement can be used. Some can augment with acetabular reinforcement ring. If the coverage of acetabular cup less than $60 \%$, structural support might needed by using cement or bone graft. There is other alternative technique to reconstruct the acetabulum at a superior location which is called high hip center. This method provides better bone implant interface which no need for bone graft. But disadvantages are limb lengthen discrepancy and proximalisation of the cup increase the shearing forces loading on the hip which may lead to early loosening. Bicanit et al. [7] stated every millimetre of proximalisation, the load on hip increses for about $0.1 \%$.

For femoral head in DDH, there are 2 main issues commonly occur. First was the orientation of femoral head and second was femoral length. In DDH, the femoral usually increase in anteversion and valgus deformity. The medullar canal usually narrower compare to normal hip. Smaller diameter femoral implant might need to prepare pre-operatively. The placement of femoral component is recommended in neutral position or slight anteversion.

Soft tissue release might helpful in reduction for low dislocation hip. In high dislocation more than $4 \mathrm{~cm}$, soft tissue release itself is not adequate and femoral osteotomy shortening is recommended. The risk of sciatic nerve injury may occur in acute reduction resulting in lengthening more than $4 \mathrm{~cm} \mathrm{[8].} \mathrm{Femoral} \mathrm{os-}$ teotomy can be divided according to their anatomy of osteotomy. Proximal or trochanteric osteotomy is commonly performed to shorten the femur and cor- 
rection of the orientation of femoral component. This procedure provide a good surgical visualization and abductor mechanism of the hip is preserved, hence provide a stable hip post operatively. But there is relatively high risk of non-union about 6\% [9]. The other method was subtrochanteric osteotomy. This allows to correct the orientation and shortening of femur without compromising the metaphyseal region, the risk of non-union also low.

\section{Conclusion}

Total hip arthroplasty in development dyplastic hip is very challenging procedure in view of abnormal anatomy and biomechanic of the hip. A proper pre-operative planning is important for successful surgery.

\section{Conflicts of Interest}

The authors declare no conflicts of interest regarding the publication of this paper.

\section{References}

[1] Boo, N.Y. and Rajaram, T. (1989) Congenital Dislocation of Hips in Malaysian Neonates. Singapore Medical Journal, 30, 368-371.

[2] Argenson, J.N., Flecher, X., Parratte, S. and Aubaniac, J.M. (2007) Anatomy of the Dysplastic Hip and Consequences for Total Hip Arthroplasty. Clinical Orthopaedics and Related Research, 465, 40-45. https://doi.org/10.1097/BLO.0b013e3181576052

[3] Sanchez-Sotelo, J., Trousdale, R.T., Berry, D.J. and Cabanela, M.E. (2002) Surgical Treatment of Developmental Dysplasia of the Hip in Adults: I. Nonarthroplasty Options. The Journal of the American Academy of Orthopaedic Surgeons, 10, 321-333. https://doi.org/10.5435/00124635-200209000-00004

[4] Liu, S., Zuo, J., Li, Z., Yang, Y., Liu, T., Xiao, J. and Gao, Z. (2016) Study of Three-Dimensional Morphology of the Proximal Femur in Developmental Adult Dysplasia of the Hip Suggests that the On-Shelf Modular Prosthesis May Not Be an Ideal Choice for Patients with Crowe Type IV Hips. International Orthopaedics, 14, $1-7$.

[5] Kim, J.S., Park, T.S., Park, S.B., Kim, J.S., Kim, I.Y. and Kim, S.I. (2000) Measurement of Femoral Neck Anteversion in 3D. Part 1: 3D Imaging Method. Medical and Biological Engineering and Computing, 38, 603-609.

https://doi.org/10.1007/BF02344864

[6] Paavilainen, T., Hoikka, V. and Solonen, K.A. (1990) Cementless Total Replacement for Severely Dysplastic or Dislocated hips. The Journal of Bone and Joint Surgery, 72, 205-211. https://doi.org/10.1302/0301-620X.72B2.2312556

[7] Eftekhar, N. (1993) Congenital Dysplasia and Dislocation. In: Eftekhar, N., Ed., Total Hip Arthroplasty, St. Louis, V. Mosby, 92.

[8] Farrell, C.M., Springer, B.D., Haidukewych, G.J. and Morrey, B.F. (2005) Motor Nerve Palsy Following Primary Total Hip Arthroplasty. Journal of Bone and Joint Surgery, 87, 2619-2625. https://doi.org/10.2106/JBJS.C.01564

[9] Kerboull, L., Hamadouche, M. and Kerboull, M. (2007) Transtrochanteric Approach to the Hip. Interactive Surgery, 2, 149-154.

https://doi.org/10.1007/s11610-007-0068-9 